readmission rate was also significantly lower (1.9\% vs. $11.8 \%$, $\mathrm{p}=0.047)$ in the MAP group compared to the non-MAP group. Conclusion: MAP program appears to improve postoperative outcomes in patients with EOC undergoing surgery by shortening hospital stay, reducing time to chemotherapy, and lowering rates of hospital readmission. Larger prospective studies are warranted to better understand the role of prehabilitation in patients of EOC.

Poster (034)

Epithelial Ovarian Cancer including Borderline Tumor https://doi.org/10.3802/jgo.2021.32.S1.034

\section{Simplified selection criteria for the secondary cytoreductive surgery in recurrent ovarian cancer}

\section{Joo-Hyuk Son," Jimin Lee, Tae-Wook Kong, Jiheum Paek, Suk-Joon Chang, Hee-Sug Ryu}

Ajou University School of Medicine, Suwon, Korea (jh920@ajou.ac.kr)

Objective: Indications for secondary cytoreductive surgery (SCS) in ovarian cancer are often dependent on multiple confounding factors. We aimed to evaluate the treatment outcomes of recurrent ovarian cancer and investigate the factors for identifying patients who could most likely benefit from SCS. Methods: We retrospectively reviewed medical records of patients with recurrent ovarian cancer from 2003 to 2020. Treatment outcomes and potential factors influencing survival were evaluated between the patients who received chemotherapy alone (chemotherapy group) and those who received SCS (surgery group) after recurrence.

Results: Altogether, 262 patients with recurrent ovarian cancer were identified. The patients' median age was $53(20-80)$ years and $87.4 \%$ of patients had initial stage III/IV disease. Eightynine $(34 \%)$ patients received SCS. The median survival was $41.0(95 \%$ confidence interval $[\mathrm{CI}]=37.4-44.5)$ months, and 88.0 (95\% CI=64.2-111.7) months in the chemotherapy and the surgery groups, respectively. Patients in the surgery group were more likely to be young and showed no gross residual disease at primary surgery, BRCA $1 / 2$ mutation, limited metastatic lesion, and low rate of ascites $(\mathrm{p}=0.001, \mathrm{p}=0.001, \mathrm{p}=0.001, \mathrm{p}=0.001$, and $\mathrm{p}=0.04$, respectively). Multivariate analysis showed limited regional carcinomatosis (single region or up to 3 regions with limited carcinomatosis) ( $\mathrm{p}=0.045$ ) as the only significant factor for predicting no residual disease after SCS. In platinumsensitive recurrent patients with limited regional recurrence, the complete resection rate was $87.6 \%$.

Conclusion: SCS showed a significant survival impact on a well- selected patient population. Limited regional recurrence can be considered simplified criteria for SCS in platinum-sensitive recurrent ovarian cancer patients.

Poster (035)

Epithelial Ovarian Cancer including Borderline Tumor https://doi.org/10.3802/jgo.2021.32.S1.035

\section{The impact of appendectomy in grossly normal appendix on the survival outcomes among mucinous borderline ovarian tumors and mucinous ovarian carcinomas, in a tertiary hospital}

\author{
Kulisara Nanthamongkolkul, Krantarat Peeyananjarassri," \\ Ingporn Jiamset, Wuttipong Chusook, Sathana Boonyapipat, \\ Rakchai Buhachat \\ Prince of Songkla University, Songkhla, Thailand (krantarat@yahoo.com)
}

Objective: To evaluate the survival outcomes of appendectomy in grossly normal appendix among patients with mucinous borderline ovarian tumors and mucinous carcinomas Methods: A retrospective cohort study was performed in Songklanagarind Hospital between 2002 and December 2019. All patients with mucinous borderline ovarian tumors and mucinous ovarian carcinomas who had grossly normal appendix were included. Overall survival (OS) and progression-free survival (PFS) were analyzed using the Kaplan-Meier method and were compared with the log-rank test. Univariate and multivariate Cox regression analysis were used to determine the independent factors associated with OS and PFS.

Results: Of 534 patients, appendectomy was performed in 344 (64\%). Of these, $6(1.7 \%)$ were primary appendiceal tumors and $6(1.7 \%)$ were appendiceal metastasis from the ovarian tumors. During the median follow up of 74.5 months, 100 deaths were identified. The OS and PFS were better in patients with appendectomy compared to non-appendectomy (5year OS, $87.9 \%$ vs. $77.9 \%, p=0.001$ and 5 -year $\mathrm{PFS}, 81.2 \%$ vs. $62.6 \%, \mathrm{p}<0.001)$. Independent factors associated with worse OS and PFS were non-appendectomy, mucinous carcinoma, and advanced International Federation of Gynaecology and Obstetrics stage. Secondary ovarian tumor was the risk factor for a worse PFS but not significant in OS. Suboptimal debulking tumor also was the risk factor for a worse OS but not significant for PFS.

Conclusion: Appendectomy in grossly normal appendix among patients with mucinous borderline ovarian tumors and mucinous ovarian carcinomas was independent prognostic factor for a better OS and PFS. 